ARTICLE

\title{
Phase diagram for the transition from photonic crystals to dielectric metamaterials
}

\author{
Mikhail V. Rybin ${ }^{1,2}$, Dmitry S. Filonov², Kirill B. Samusev,2, Pavel A. Belov2, Yuri S. Kivshar 2,3
}

$\&$ Mikhail F. Limonov ${ }^{1,2}$

Photonic crystals and dielectric metamaterials represent two different classes of artificial media but are often composed of similar structural elements. The question is how to distinguish these two types of periodic structures when their parameters, such as permittivity and lattice constant, vary continuously. Here we discuss transition between photonic crystals and dielectric metamaterials and introduce the concept of a phase diagram, based on the physics of Mie and Bragg resonances. We show that a periodic photonic structure transforms into a metamaterial when the Mie gap opens up below the lowest Bragg bandgap where the homogenization approach can be justified and the effective permeability becomes negative. Our theoretical approach is confirmed by microwave experiments for a metacrystal composed of tubes filled with heated water. This analysis yields deep insight into the properties of periodic structures, and provides a useful tool for designing different classes of electromagnetic materials with variable parameters.

\footnotetext{
${ }^{1}$ Ioffe Institute, St Petersburg 194021, Russia. ${ }^{2}$ Department of Nanophotonics and Metamaterials, ITMO University, St Petersburg 197101, Russia. ${ }^{3}$ Nonlinear Physics Center and the ARC Center of Excellence CUDOS, Australian National University, Canberra Australian Capital Territory 0200, Australia. Correspondence and requests for materials should be addressed to M.V.R. (email: m.rybin@mail.ioffe.ru) or to Y.S.K. (email: ysk@internode.on.net).
} 
T he concepts of phase transitions and phase diagrams are among the most fundamental concepts in physics. Here we reveal and analyse a rather unusual type of phase transitions between two classes of artificial electromagnetic structures: photonic crystals $(\mathrm{PhCs})^{1,2}$ and metamaterials $(\mathrm{MMs})^{3,4}$. Rapidly growing attention to the study of PhCs and MMs is due to their unique electromagnetic properties and the exciting new physics these structures may offer for novel applications in photonics ${ }^{5-9}$. The resonant properties of $\mathrm{PhCs}$ are driven by the familiar Bragg scattering from a periodic structure when the lattice constant becomes comparable with the wavelength of light. MMs are regarded as effective media with averaged parameters which in turn can be engineered by changing the properties of their subwavelength elements modifying the propagation of electromagnetic waves with the wavelengths much larger than the lattice constant.

Many properties of conventional MMs are usually defined by the optically induced magnetic response of metallic wires or splitring resonators, which has been demonstrated experimentally in many studies for both microwave and near-infrared spectral regions ${ }^{10,11}$. However, the scaling of such MMs to higher frequencies encounters many problems, including strong absorption of electromagnetic radiation in metals. Recently suggested electromagnetic periodic structures composed of high-permittivity dielectric elements ${ }^{12-14}$ can avoid these problems, while still delivering an effective magnetic response. The main physics underlying the properties of such all-dielectric MMs is based on the resonant modes of the Mie scattering ${ }^{15}$ supported by dielectric particles ${ }^{16,17}$. Mie resonances provide a mechanism for the generation of an optically induced resonant magnetic response based on the displacement current, and they offer a simpler and more versatile route towards the fabrication of isotropic MMs and metasurfaces operating at optical frequencies.

PhCs and all-dielectric MMs can be composed of the similar structural elements arranged in the similar geometries. Such structures offer a unique opportunity to study a transition from $\mathrm{PhCs}$ to $\mathrm{MMs}$ when their parameters, such as dielectric permittivity and lattice period, vary continuously. The transition we describe here can be mapped onto a phase diagram; however, it is quite different from phase transitions well known in other fields such as thermodynamics. The thermodynamical phase transitions appear as transformations of a system from one state or 'phase' to another with changing external variables such as pressure, temperature, chemical potential and so on. Here we reveal and analyse a rather unusual type of phase transitions between two regimes of light propagation, that is, the regime where the propagation of light is mainly influenced by the interference between multiple Bragg scattered (PhC 'phase'), and the regime where the propagation of light is mainly determined by the properties of each single element of the materials (MM 'phase').

First, we identify two different scenarios for the transition of a two-dimensional (2D) square lattice of dielectric rods from $\mathrm{PhC}$ to MM (Fig. 1). The first scenario that has never been discussed previously occurs when the lattice constant $a$ decreases in comparison with the fixed radius of rods $r$ and dielectric permittivity $\varepsilon$. This transformation leads to an increase of the filling ratio $r / a$, and it makes possible the homogenization ${ }^{18}$ of the periodic dielectric structure with the negative effective permeability $(\mu<0)$ at higher values of $\varepsilon$ (ref. 19-23).

The second scenario occurs when the dielectric permittivity of rods $\varepsilon$ varies in a fixed square lattice. This transformation leads to an increase of the Mie scattering wavelength, and it can also provide the conditions for the homogenization approach with negative $\mu$. While photonic structures with varying $\varepsilon$ were studied earlier theoretically ${ }^{24,25}$, the transition between PhCs and MMs

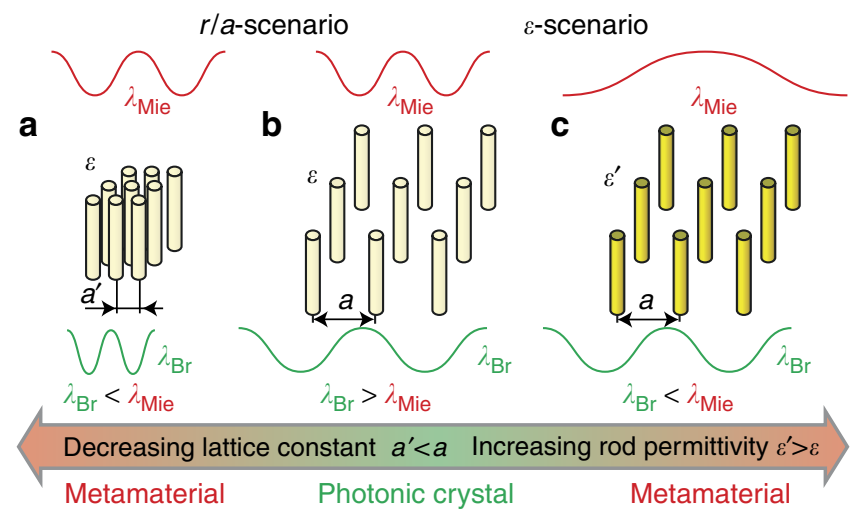

Figure 1 | Transition from photonic crystals to metamaterials. Schematic illustrates two possible transformations of the $\mathrm{PhC}(\mathbf{b})$ to $\mathrm{MM}(\mathbf{a}, \mathbf{c})$ through the competition between the Bragg and Mie scattering wavelengths. To satisfy the classical homogenization condition in the low-frequency longwavelength limit $\left(\lambda_{\text {Bragg }}<\lambda_{\text {Mie }}\right.$ ), one may decrease the lattice constant and increase the filling ratio $r / a(r / a$-scenario) or, alternatively, increase the rod permittivity $\varepsilon$ ( $\varepsilon$-scenario).

has never been analysed previously to our best knowledge, especially in terms of the phase diagram, as discussed below.

We notice that the aim of both scenarios is the same: to tune the wavelength of the Mie mode $\lambda_{\text {Mie }}$ higher than the Bragg wavelength $\lambda_{\mathrm{Br}}$ that can be achieved by either decreasing the Bragg wavelength or by increasing the Mie wavelength. Decreasing the Bragg wavelength means decreasing the lattice constant (building block size) $a$, which will be smaller than the operating wavelength for the MM behaviour, $\lambda_{\text {Mie }}$.

Here we integrate both scenarios and introduce a novel concept of a phase transition between all-dielectric $\mathrm{PhC}$ and $\mathrm{MM}$ and suggest the corresponding phase diagram. The phase diagram $\mathrm{PhC}-\mathrm{MM}$ is represented on the plane 'filling ratio versus dielectric permittivity' for an example of 2D structure composed of dielectric circular rods. Our theory is confirmed by very careful experimental design and analysis.

\section{Results}

An interplay between Bragg and Mie resonances. To analyse the $\mathrm{PhC}-\mathrm{MM}$ phase transition, we investigate theoretically electromagnetic properties of the dielectric periodic medium depending on the dielectric contrast, filling ratio and incident wave polarization. As an illustrative example, we consider here 2D square lattices of parallel dielectric circular rods infinitely long in the $z$ direction with the radius $r$ and real frequency independent permittivity $\varepsilon$. The rods are embedded in air $\left(\varepsilon_{\text {air }}=1\right)$ and the arrays can be characterized by the filling ratio $r / a$. We have derived three key sets of spectroscopic data, namely: (i) spectra of the Mie scattering by an isolated rod; (ii) photonic band structure of an infinite 2D square lattice composed of rods; and (iii) transmittance of a 2D square lattice of 10 layers in length. All data sets have been calculated for a wide range of the rod permittivity $1 \leq \varepsilon \leq 100$ with the step of $\Delta \varepsilon=1$ ( $\varepsilon$-scenario) and in the range of filling ratio $0 \leq r / a \leq 0.5$ with the step of 0.01 ( $r / a$-scenario). In the $r / a$-scenario cylinder radius is supposed to be as a fixed parameter. Note that at $r / a=0.5$ all rods touch each other and at $r / a>0.5$ the rods penetrate into each other and the structure appears as inverted one of air holes in a dielectric matrix.

First, we consider the Mie scattering by an isolated rod. The far-field scattering can be expanded into orthogonal electromagnetic dipolar and multipolar modes, described by circular Lorenz-Mie resonant coefficients $a_{n}$ (transverse-electric 
(TE) polarization) and $b_{n}$ (transverse-magnetic (TM) polarization) corresponding to electric and magnetic moments, respectively ${ }^{15}$. Figure 2 presents the numerically calculated spectra of the Mie scattering efficiency $Q_{\text {sca }, n}=\frac{2}{x}\left|a_{n}\right|^{2}$ (ref. 26) for the dipole $\mathrm{TE}_{0}$ and multipole $\mathrm{TE}_{n}(n \geq 1)$ modes in the range of dimensionless frequencies $a / \lambda$ from 0 to 0.8 . Each mode consists of an infinite set of quasi-equidistant modes $\mathrm{TE}_{n k}$ (ref. 27). The Mie modes of the rod are denoted as $\mathrm{TE}_{n k}$, where $n$ is integer related to the symmetry of the mode, and $k \geq 1$ identifies resonance number for the modes with fixed symmetry $n$. The calculations reveal a strong decrease of the Mie eigenfrequencies and narrowing of the resonant Mie bands with $\varepsilon$ increasing, as can be clearly seen for the lowest $\mathrm{TE}_{01}$ magnetic Mie resonance in Fig. 2.

The photonic band structures for the $2 \mathrm{D}$ square lattice of circular rods were computed numerically using the plane wave expansion method ${ }^{28}$. The square $2 \mathrm{D}$ lattice has a square Brillouin zone, with the three special points $\Gamma, \mathrm{X}$ and $\mathrm{M}$ corresponding to $\mathbf{k}=0, \mathbf{k}=\frac{\pi}{a} \hat{x}$, and $\mathbf{k}=\frac{\pi}{a} \hat{x}+\frac{\pi}{a} \hat{y}$, respectively. We restrict here our discussion to the case for which the wave vector $\mathbf{k}$ of the eigenmodes is oriented in the $\Gamma \rightarrow \mathrm{X}$ direction of the first Brillouin zone displaying the lowest Bragg frequencies in comparison with the $\Gamma \rightarrow \mathrm{M}$ direction (Fig. 2). The photonic band structures were obtained with $16,384(128 \times 128)$ plane waves.
The transmission spectra were calculated using the CST Microwave Studio software for the wave vector of the incident beam parallel to the $\Gamma \rightarrow \mathrm{X}$ direction. We considered the $2 \mathrm{D}$ square lattice with different thicknesses along $x$ axis that leads to creation of the photonic bandgaps, including thicknesses of 10 lattice layers as shown in Fig. 2.

The calculated data allow us to analyse precisely the evolution of the low-frequency region of the photonic band structure formed by a complicated mixture of the dispersion curves originating from Mie and Bragg resonances and propagating modes. Since our goal is to discuss the $\mathrm{PhC}-\mathrm{MM}$ transition, we concentrate on the $\mathrm{TE}$ polarization where the lowest $\mathrm{TE}_{01}$ magnetic dipole resonance of the rods results in a negative magnetic permeability in the Mie gap spectral region ${ }^{19}$.

The physics of the PhC-MM transition is displayed by the gap map (Fig. 3) that relates the Bragg and Mie gaps positions to the rod permittivity $\varepsilon$ for given $r / a=0.25$. These illustrate essential features such as strong decreasing of Mie resonance frequencies with $\varepsilon$ increase followed by intersection of the Mie and Bragg bands. The Mie resonances of individual rods form both non-dispersive flat bands in the photonic structure and Mie photonic gaps in the energy spectrum (Figs 2 and 3). For low-contrast PhCs, all Mie resonances are located is a frequency range higher than the lower Bragg gap. With increasing rod
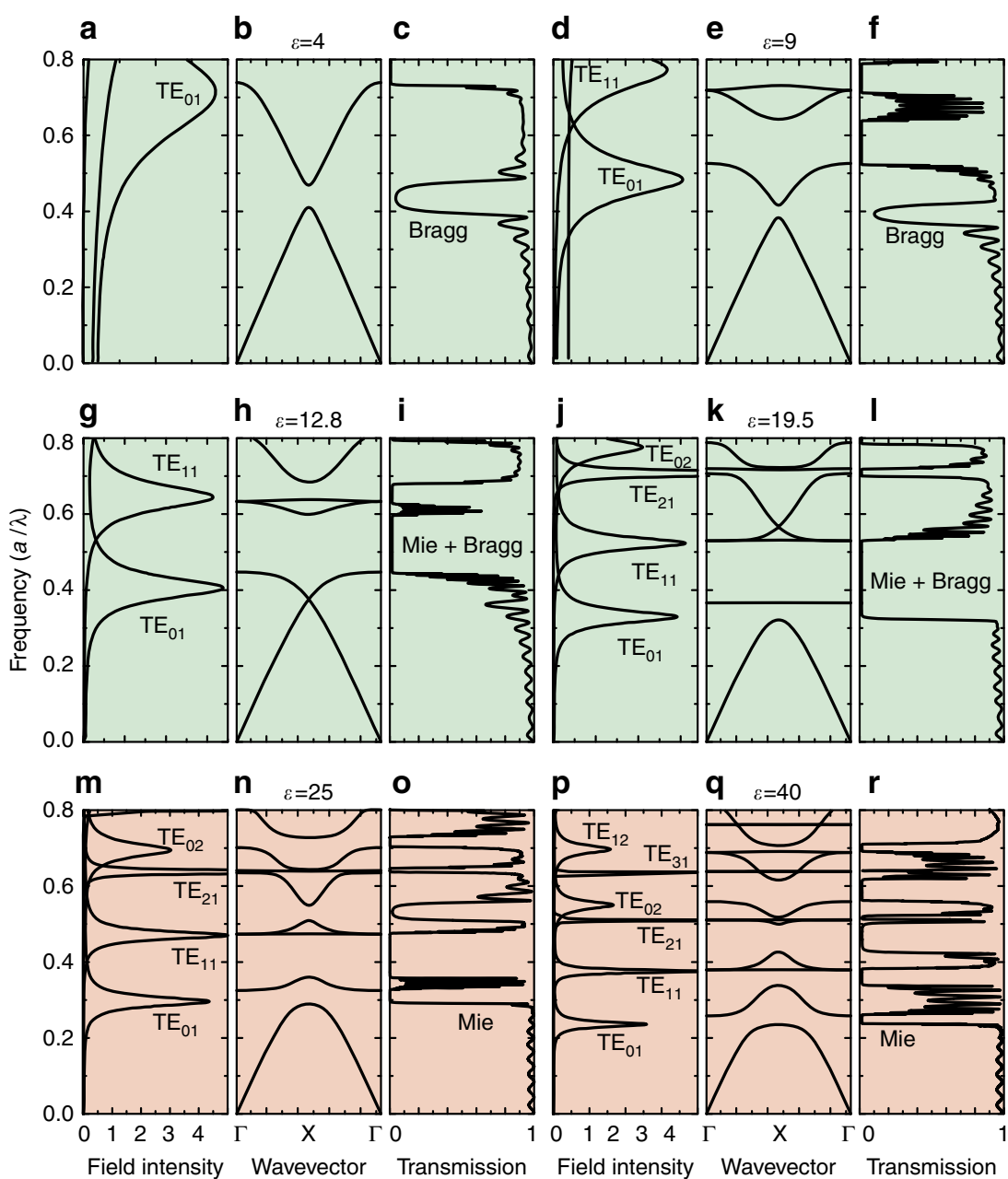

Figure 2 | Numerical calculations. $(\mathbf{a}),(\mathbf{d}),(\mathbf{g}),(\mathbf{j}),(\mathbf{m}),(\mathbf{p})$ Calculated Mie scattering efficiency $Q_{s c a, n}$ for an isolated dielectric circular rod for $T E_{n}(n \geq 0)$ modes. (b), (e), (h), (k), (n), (q) The band structure for 2D square lattice of rods with $r=0.25 a$ in air $\left(\varepsilon_{\text {air }}=1\right)$ for the TE polarization. The band structure is shown between the $\Gamma$ point (wave vector $\mathbf{k}=0$ ) and the $\mathrm{X}$ point $(|\mathbf{k}|=\pi / a$ along the $x$ direction). ((c), (f), (i), (I), (o), ( $\mathbf{r})$ The transmittance calculated for 10 lattice layers of the 2D square structure of rods in air for the TE polarization. The frequency and wave vector are plotted in dimensionless units $a / \lambda$, where $\lambda$ is vacuum wavelength and $a$ denotes the lattice constant. (a)-(c) $\varepsilon=4,(\mathbf{d})-(\mathbf{f}) \varepsilon=9,(\mathbf{g})-(\mathbf{i}) \varepsilon=12.8,(\mathbf{j})-(\mathbf{I}) \varepsilon=19.5,(\mathbf{m})-(\mathbf{o}) \varepsilon=25$, (p)-(r) $\varepsilon=40$. 


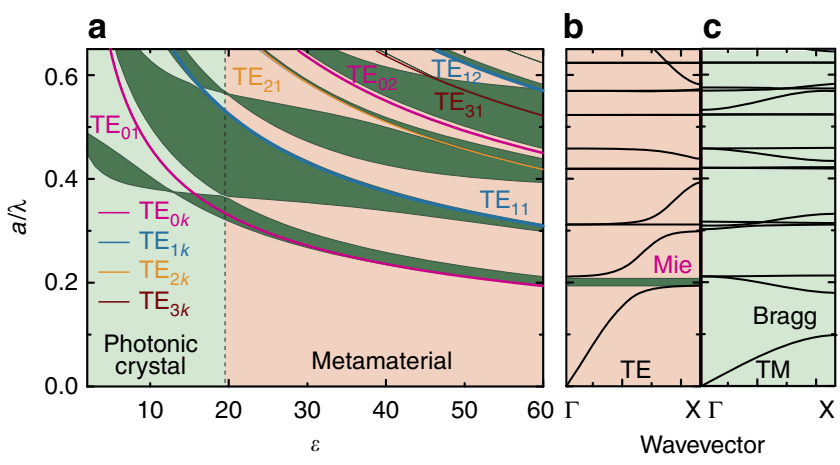

Figure 3 | Bandgap diagram for the 2D square lattice of circular rods. (a) The bandgap diagram obtained from the photonic band structures calculated for $1 \leq \varepsilon \leq 60$ with steps of $\Delta \varepsilon=1$. The rods are embedded in air, $r / a=0.25$, and TE polarization is considered. The Bragg and Mie gaps obtained from the band structure calculations are marked by dark green. $(\mathbf{b}, \mathbf{c})$ The photonic band structure for the 2D square lattice of rods with $\varepsilon=60$ for TE and TM polarization, respectively, for the $\Gamma \rightarrow \mathrm{X}$ scan of the wave vector $\mathbf{k}$.

permittivity $\varepsilon$, the Mie resonances of single rods and correspondingly Mie bands of the structure demonstrate a shift from the higher to low frequencies, crossing the strongly dispersive Bragg bands. The creation of the lowest Mie gap driving the artificial magnetism is clearly seen both in the band structure and in the transmission spectra (Figs 2 and 3).

Two crossover scenarios for a metacrystal. Armed with the results of our calculations, we verify our concept experimentally by engineering a metacrystal composed of plastic circular tubes filled with water and forming a $2 \mathrm{D}$ square lattice with variable lattice constant $a$ (Fig. 4). The structure of the metacrystal enables us to perform two types of experiments according to the $r / a$ - and $\varepsilon$-scenarios. The $r / a$-scenario was realized using a special construction of the metacrystal that makes it possible to change the distance between tubes synchronously in $x$ and $y$ directions keeping the $2 \mathrm{D}$ square symmetry of the lattice (Fig. 4). For the $\varepsilon$-scenario, we employ the advantages of strong temperature dependence of the dielectric permittivity of water that is characterized by $\varepsilon=80$ at $20^{\circ} \mathrm{C}$ and $\varepsilon=50$ at $90^{\circ} \mathrm{C}$ in the microwave frequencies range from 1 to $6 \mathrm{GHz}$ (refs 29,30). On the basis of the calculated results and the parameters of the metacrystal, we chose intervals of most interest for each scenario, namely $60 \leq \varepsilon \leq 80$ at $0.7 \leq r / a \leq 0.8$ for the $\varepsilon$-scenario and $0.65 \leq r / a \leq 1.9$ at $\varepsilon=60$ for the $r / a$-scenario.

The transmission spectra of the metacrystal installed in an anechoic chamber were measured in the $1-3-\mathrm{GHz}$ range. A rectangular horn antenna (TRIM $0.75-18 \mathrm{GHz}$; DR) connected to a transmitting port of the vector network analyser Agilent E8362C was used to approximate plane wave excitation. A similar horn antenna was employed as a receiver. For the electromagnetic wave incident perpendicular to the tube axis $z$, the two transverse polarizations appear decoupled and the vector wave problem can be reduced to two independent scalar equations. As a result, the modes of $2 \mathrm{D}$ PhCs can be classified as either TE modes $\left(E_{x}, E_{y}\right.$ and $H_{z}$ ) with the electric field confined to the $x-y$ plane and the magnetic field polarized along the axes of the rods (Fig. 4) or TM modes $\left(H_{x}, H_{y}\right.$ and $\left.E_{z}\right)$ for which the magnetic field is oriented perpendicular to the rod axis $z$. For the TM polarization, such structures can not show any magnetic activity ${ }^{31}$, so we only consider TE polarization.

Figure 5 demonstrates excellent agreement between the measured and calculated transmission spectra both for $r / a$ and

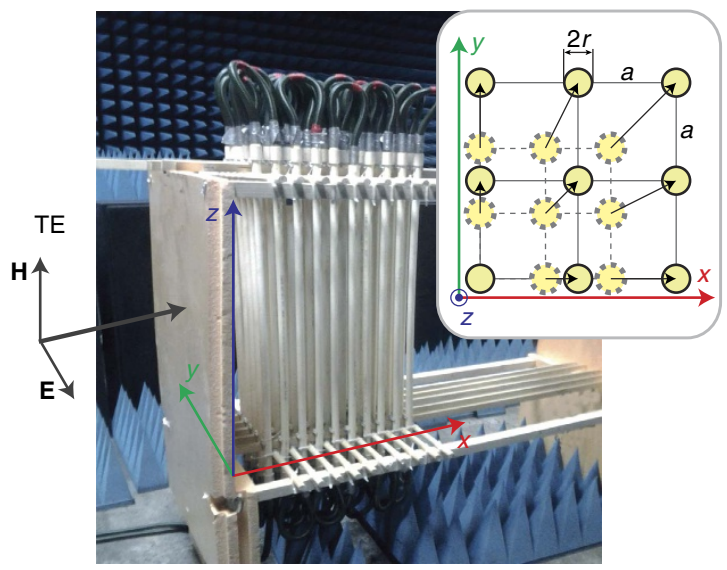

Figure 4 | Experimental set-up. An anechoic chamber with a metacrystal composed of 10 tubes in length ( $x$ axis) and 5 tubes in width ( $y$ axis), 50 tubes in total. Each tube has a length of $1 \mathrm{~m}$ (along $z$ axis), an outer radius of $2 \mathrm{~cm}$ and an inner radius of $1.7 \mathrm{~cm}$. All tubes are connected in a closed series circuit together with a water heater and a temperature stabilizer. Insert: schematic illustration of the transformation of the 2D square lattice composed of dielectric rods as the lattice constant $a$ increase. The scheme shows the square lattice from above. The rods with dielectric permittivity $\varepsilon$ and fixed radius $r$ are embedded in air $\left(\varepsilon_{\text {air }}=1\right)$. The material is homogeneous along the $z$ direction and periodic along $x$ and $y$.

$\varepsilon$ dependencies. For the $r / a$-scenario, we started our experiment from the PhC phase $(r / a=0.08)$ and compressed the structure by decreasing the lattice constant $a$ (Fig. 5a). The lowest Bragg gap demonstrates remarkable shift towards high-frequency region, then nearly almost disappears at $r / a \approx 0.09$ when it approaches the unchanging $\mathrm{TE}_{01}$ Mie gap from the low-frequency side, and finally appears again at $r / a \approx 0.095$ on the high-frequency side of the Mie gap. As a result, the lowest Bragg band and the $\mathrm{TE}_{01} \mathrm{Mie}$ band change their positions in the energy scale inaugurating the transition into MM phase. In contrast, for the $\varepsilon$-scenario, the Bragg gap position changes very slowly as opposed to the strong shift of the $\mathrm{TE}_{01}$ Mie band that finally leads to the same general result (Fig. 5d).

\section{Discussion}

Figure 6a shows our main result: the $\mathrm{PhC}-\mathrm{MM}$ phase diagram for the $2 \mathrm{D}$ square lattice of circular rods. The axes represent the dielectric permittivity $\varepsilon$ and filling ratio $r / a$. The phase diagram was obtained based on 50 gap maps (such as in Fig. 3a) calculated in the permittivity region $1 \leq \varepsilon \leq 100$ with the step $r / a=0.01$ for TE polarization. The diagram is divided into two areas, which represent the $\mathrm{PhC}$ and $\mathrm{MM}$ phases. The transition from $\mathrm{PhC}$ phase to MM phase can be identified by critical points, which are defined by a special combination of permittivity $\varepsilon$ and filling ratio $r / a$. The curvature of the second dispersion curve around the $\Gamma$ point decreases and finally becomes perfectly flat along the $\Gamma X$ path (at $\varepsilon=19.5, r / a=0.25$, Fig. $2 \mathrm{k}$ ). With future increase in $\varepsilon$ or change in $r / a$, the $\mathrm{TE}_{01}$ Mie gap completely splits from complicated coupled Mie-Bragg band becoming the lowest gap in the spectrum and the MM phase appears. Within this Mie gap, the magnetic permeability is negative and all waves in the medium are evanescent. Since all the Bragg gaps are located at higher frequencies, any diffraction losses in the vicinity of the Mie resonance are absent. The transition from the $\mathrm{PhC}$ phase to the MM phase is completed and the medium is classified as the MM with $\mu<0$. A phase transition is usually characterized by an order parameter that basically arises from symmetry breaking but can also be defined for non-symmetry-breaking transitions ${ }^{32}$. Here 

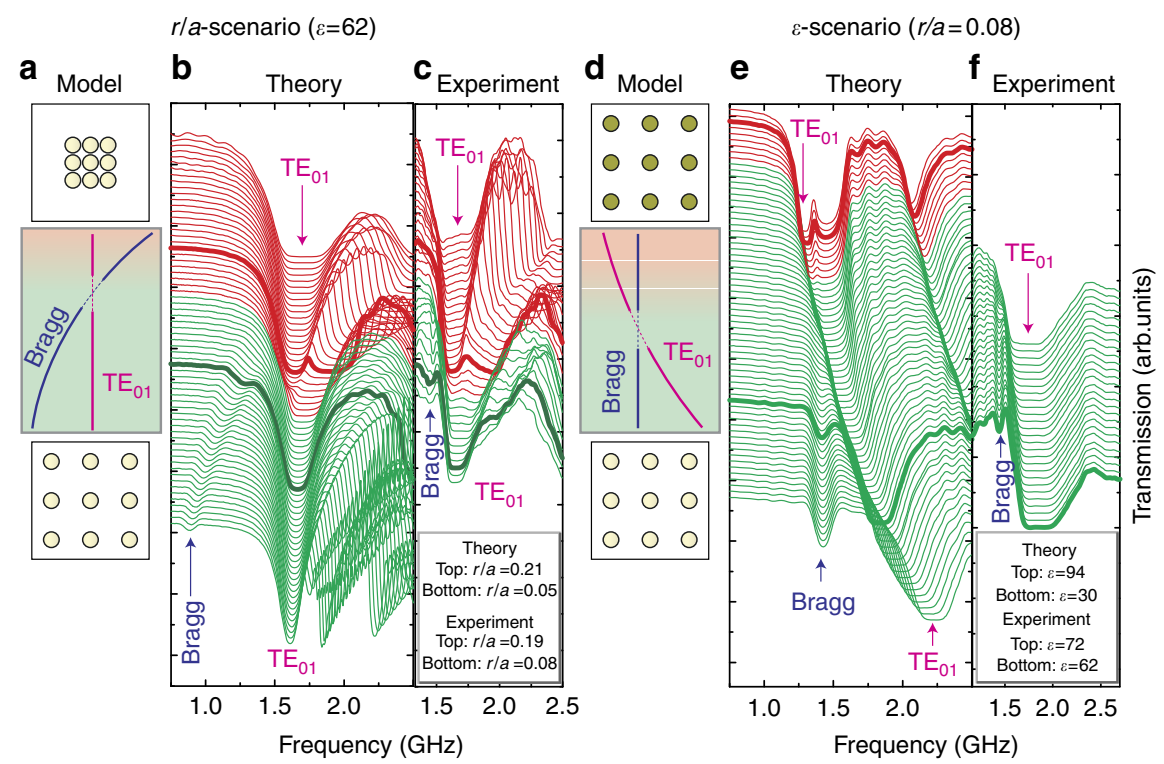

Figure 5 | Transmission spectra for a square lattice of circular rods. TE polarization. (a-c) $\mathrm{r} / \mathrm{a}$-scenario, $\varepsilon=62$. The calculated (b) and experimentally measured (c) transmission spectra of the metacrystal as a function of the filling ratio $r / a$. The calculated spectra are shown in the range $0.05 \leq r / a \leq 0.21$, the experimental spectra are shown in the range $0.08 \leq r / a \leq 0.19$. (d-f) $\varepsilon$-scenario, $r / a=0.08$. The calculated (e) and experimentally measured (f) transmission spectra of the metacrystal as a function of the dielectric permittivity. The calculated spectra are shown in the range $30 \leq \varepsilon \leq 94$, the experimental spectra are shown in the range $62 \leq \varepsilon \leq 94$. The spectra are shifted vertically by the constant value.
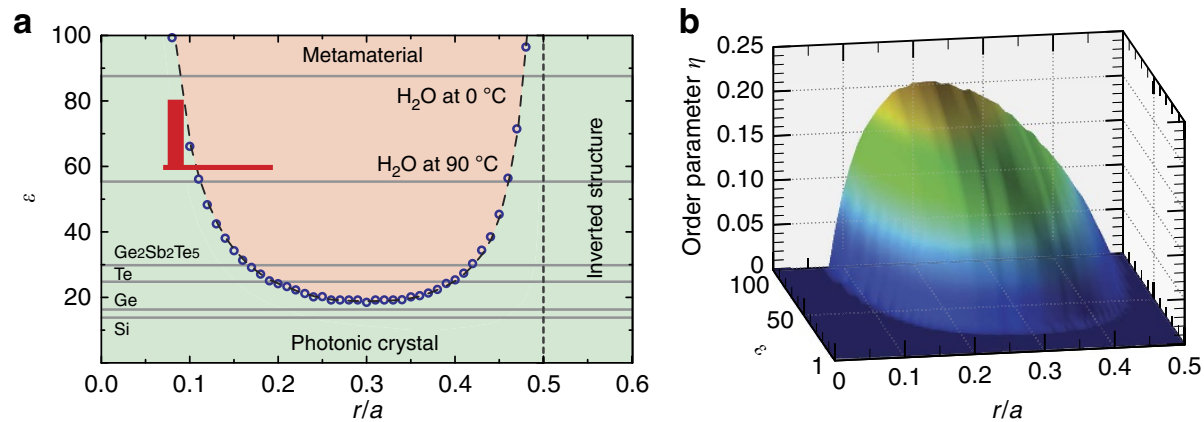

Figure 6 | PhC-MM phase diagram. (a) PhC-MM phase diagram for a square lattice of dielectric circular rods. TE polarization. Blue circles indicate the points where the $\mathrm{TE}_{01}$ Mie gap splits from Bragg band becoming the lowest gap in the spectrum (Fig. 2). The experimentally investigated regions are marked by red. The dielectric permittivities $\varepsilon$ of different materials are marked by horizontal lines. (b) The dependence of the order parameter $\eta$ on the dielectric permittivity $\varepsilon$ and the filling factor $r / a$. The order parameter is defined as a difference in the energy between the $\mathrm{TE}_{01}$ Mie and Bragg gaps in the MM phase.

for the unusual phase transition $\mathrm{PhC} \rightarrow \mathrm{MM}$, we define the order parameter $\eta$ as a measure of splitting in the energy scale between the $\mathrm{TE}_{01}$ Mie and Bragg gaps. It is zero in the PhC phase and nonzero in the MM phase. Figure $6 \mathrm{~b}$ presents the order parameter depending on both external variables $\varepsilon$ and $r / a$.

Now, we review some features of the $\mathrm{PhC}-\mathrm{MM}$ phase diagram. At low filling ratio $r / a<0.05$, the concentration of the completely isolated rods in the lattice is too small for achieving negative average permeability at reasonable values of $\varepsilon$. In contrast, when distance between rods become very small $(r / a>0.45)$, the inter-rod interaction become strong and the hopping of the Mie photons from one rod to another dominates. This means that Mie resonances are not localized anymore and they finally exhibit the character of propagating Bloch waves. All requirements for the appearance of the MM phase are broken and the $\mathrm{PhC}$ phase extends its region of existence to very high $\varepsilon$. We notice that for the metacrystals with a small lattice constant $a$, the frequency of the Mie resonance is increasing. Therefore, there is no transition to the MM phase in this limit.
Identification of candidates for dielectric low-loss materials is critical for designing a practical MM for applications in different ranges of the electromagnetic spectrum. As an example, in Fig. 6a, we mark the regions of the MM phase for water in the microwave range (region $55 \leq \varepsilon \leq 87)^{31}$, the chalcogenide glass $\mathrm{Ge}_{2} \mathrm{Sb}_{2} \mathrm{Te}_{5}$ $(\varepsilon \approx 30)^{33}$ and tellurium $(\varepsilon \approx 25)^{34}$ in infrared. Thus, the well-studied $2 \mathrm{D}$ square lattice of germanium $(\varepsilon \approx 16)$ and silicon $(\varepsilon \approx 13)^{34}$ rods has only PhC phase.

\section{Conclusion}

We have studied a metacrystal composed of dielectric circular rods and introduced the concept of a phase diagram in the permittivity-filling ratio plane. The boundaries of the MM phase have been obtained theoretically and confirmed experimentally. On the basis of the proposed approach, one can obtain different $\mathrm{PhC}-\mathrm{MM}$ phase diagrams by altering the dimension, symmetry, composition, size and geometry of the structural elements within a unit cell. The underlying mechanism of the PhC-MM transition provides unique criteria for goal-oriented search of novel low-loss 
MM with a wide range of applications at infrared and visible frequencies.

\section{References}

1. Joannopoulos, J. D., Johnson, S. G., Winn, J. N. \& Meade, R. D. Photonic Crystals: Molding the Flow of Light 2nd edn (Princeton Univ. Press, 2008).

2. Limonov, M. F. \& De La Rue, R. M. (eds) Optical Properties of Photonic Structures: Interplay of Order and Disorder (CRC Press, Taylor \& Francis Group, 2012).

3. Enghata, N. \& Ziolkowski, R. (eds) Electromagnetic Metamaterials: Physics and Engineering Exploration (Wiley-IEEE Press, 2006).

4. Smith, D. \& Liu, R. (eds) Metamaterials: Theory, Design, and Applications (Springer, 2010).

5. Cai, W., Chettiar, U. K., Kildishev, A. V. \& Shalaev, V. M. Optical cloaking with metamaterials. Nat. Photon. 1, 224-227 (2007).

6. Lapine, M., Shadrivov, I. V., Powell, D. A. \& Kivshar, Y. S. Magnetoelastic metamaterials. Nat. Mater. 11, 30-33 (2012).

7. Silva, A. et al. Performing mathematical operations with metamaterials. Science 343, 160-163 (2014).

8. Smith, D. R., Pendry, J. B. \& Wiltshire, M. C. K. Metamaterials and negative refractive index. Science 305, 788-792 (2004).

9. Soukoulis, C. M., Kafesaki, M. \& Economou, E. N. Negative-index materials: New frontiers in optics. Adv. Mater. 18, 1941-1952 (2006).

10. Soukoulis, C. M. \& Wegener, M. Past achievements and future challenges in the development of three-dimensional photonic metamaterials. Nat. Photon. 5, 523-530 (2011).

11. Zheludev, N. I. \& Kivshar, Y. S. From metamaterials to metadevices. Nat. Mater. 11, 917-924 (2012).

12. Huang, K. C., Povinelli, M. \& Joannopoulos, J. D. Negative effective permeability in polaritonic photonic crystals. Appl. Phys. Lett. 85, 543-545 (2004).

13. Kallos, E., Chremmos, I. \& Yannopapas, V. Resonance properties of optical alldielectric metamaterials using two-dimensional multipole expansion. Phys. Rev. B 86, 245108 (2012)

14. Moitra, P. et al. Realization of an all-dielectric zero-index optical metamaterial. Nat. Photon. 7, 791-795 (2013).

15. Bohren, C. F. \& Huffman, D. R. Absorption and Scattering of Light by Small Particles (Wiley-VCH, 1998).

16. Ginn, J. C. et al. Realizing optical magnetism from dielectric metamaterials. Phys. Rev. Lett. 108, 097402 (2012).

17. Zhao, Q., Zhou, J., Zhang, F. \& Lippens, D. Mie resonance-based dielectric metamaterials. Mater. Today 12, 60-69 (2009).

18. Andryieuski, A. et al. Homogenization of resonant chiral metamaterials. Phys. Rev. B 82, 235107 (2010).

19. O'Brien, S. \& Pendry, J. B. Photonic band-gap effects and magnetic activity in dielectric composites. J. Phys. Condens. Matter 14, 4035 (2002).

20. Yannopapas, V. \& Moroz, A. Negative refractive index metamaterials from inherently non-magnetic materials for deep infrared to terahertz frequency ranges. J. Phys. Condens. Matter 17, 3717 (2005).

21. Wheeler, M. S., Aitchison, J. S. \& Mojahedi, M. Three-dimensional array of dielectric spheres with an isotropic negative permeability at infrared frequencies. Phys. Rev. B 72, 193103 (2005).

22. Schuller, J. A., Zia, R., Taubner, T. \& Brongersma, M. L. Dielectric metamaterials based on electric and magnetic resonances of silicon carbide particles. Phys. Rev. Lett. 99, 107401 (2007).

23. Vynck, K. et al. All-dielectric rod-type metamaterials at optical frequencies. Phys. Rev. Lett. 102, 133901 (2009).

24. Foteinopoulou, S. Photonic crystals as metamaterials. Physica B 407, 4056 (2012).
25. Notomi, M. Theory of light propagation in strongly modulated photonic crystals: Refractionlike behaviour in the vicinity of the photonic band gap. Phys. Rev. B 62, 10696 (2000).

26. Rybin, M. V., Filonov, D. S., Belov, P. A., Kivshar, Y. S. \& Limonov, M. F. Switching from visibility to invisibility via Fano resonances: theory and experiment. Sci. Rep. 5, 8774 (2015).

27. Rybin, M. V. et al. Mie scattering as a cascade of Fano resonances. Opt. Express 21, 30107-30113 (2013).

28. Johnson, S. G. \& Joannopoulos, J. D. Block-iterative frequency-domain methods for Maxwell's equations in a planewave basis. Opt. Express 8, 173 (2001).

29. Kaatze, U. Complex permittivity of water as a function of frequency and temperature. J. Chem. Eng. Data 34, 371-374 (1989).

30. Andryieuski, A., Kuznetsova, S. M., Zhukovsky, S. V., Kivshar, Y. S. \& Lavrinenko, A. V. Water: Promising opportunities for tunable all-dielectric electromagnetic metamaterials. Sci. Rep. 5, 13535 (2015).

31. Ramakrishna, S. A. Physics of negative refractive index materials. Rep. Prog. Phys 68, 449 (2005).

32. Gooding, R., Ye, Y., Chan, C., Ho, K. \& Harmon, B. Role of non-symmetrybreaking order parameters in determining the martensitic energy barrier: The bcc-to-9R transformation. Phys. Rev. B 43, 13626 (1991).

33. Nčmec, P. et al. Ge-Sb-Te thin films deposited by pulsed laser: An ellipsometry and Raman scattering spectroscopy study. J. Appl. Phys. 106, 103509 (2009).

34. Palik, E. D. Handbook of Optical Constants of Solids vol. 3 (Academic press, 1998).

\section{Acknowledgements}

We acknowledge fruitful discussions with many of our colleagues, including W. Barnes, I. Brener, S. Fan, S. Foteinopoulou, A. Khanikaev, D.A. Powell, J.R. Sambles and A.P. Slobozhanyuk, as well as we thank S.B. Glybovski for the technical help. M.R., K.S., and M.L. acknowledge support by the Russian Foundation for Basic Research (grant 15-02-07529, theory and numerical analysis), P.B. and D.F. acknowledges support by the Russian Science Foundation (Grant 15-19-30023, experimental studies), and Y.K. acknowledges a support by the Australian Research Council.

\section{Author contributions}

M.V.R. and K.B.S. developed the theoretical model and conducted simulations and data analysis. D.S.F. performed experimental measurements. M.F.L, P.A.B. and Y.S.K. provided general guidance to the theory, numerical analysis and experiment. All authors discussed the results and contributed to the writing of the manuscript.

\section{Additional information}

Competing financial interests: The authors declare no competing financial interests.

Reprints and permission information is available online at http://npg.nature.com/ reprintsandpermissions/

How to cite this article: Rybin, M. V. et al. Phase diagram for the transition from photonic crystals to dielectric metamaterials. Nat. Commun. 6:10102 doi: 10.1038/ncomms10102 (2015).

(c) (i) This work is licensed under a Creative Commons Attribution 4.0 International License. The images or other third party material in this article are included in the article's Creative Commons license, unless indicated otherwise in the credit line; if the material is not included under the Creative Commons license, users will need to obtain permission from the license holder to reproduce the material. To view a copy of this license, visit http://creativecommons.org/licenses/by/4.0/ 